\title{
A mixture representation of the Linnik distribution
}

\author{
Samuel Kotz ${ }^{\mathrm{a}}$, I.V. Ostrovskii ${ }^{\mathrm{b}, *}$ \\ ${ }^{a}$ University of Maryland at College Park, Department of Management Science and Statistics, College Park, MD 20742, USA \\ ${ }^{\mathrm{b}}$ Bilkent University at Ankara, Department of Mathematics, 06533 Bilkent, Ankara, Turkey, and Institute for Low Temperature Physics and \\ Engineering at Kharkov, 310164 Kharkov, Ukraine
}

Received April 1994; revised October 1994

\section{Abstract}

Linnik distribution with the characteristic function

$$
\varphi_{x}(t)=1 /\left(1+|t|^{\alpha}\right), \quad 0<\alpha<2
$$

is shown to possess the following property.

Let $X_{x}, X_{\beta}$ be random variables possessing the Linnik distribution with parameters $\alpha$ and $\beta$ respectively $(0<\alpha<\beta \leqslant 2)$. Denote by $Y_{\alpha \beta}$ an independent of $X_{\beta}$ non-negative random variable with the density

$$
g(s ; \alpha, \beta)=\left(\frac{\beta}{\pi} \sin \frac{\pi \alpha}{\beta}\right) \frac{s^{\alpha-1}}{1+s^{2 \alpha}+2 s^{\alpha} \cos \frac{\pi \alpha}{\beta}}, \quad 0<s<\infty .
$$

Then

$$
X_{\alpha} \doteq X_{\beta} Y_{\alpha \beta}
$$

where $\doteq$ denotes the equality in the sense of distributions.

Infinite divisibility of mixtures of Linnik distributions with respect to the parameter $\alpha$ and scale is obtained as a corollary.

AMS 1980 Subject Classification: Primary 62H05, 60E10; Secondary 33A40

Keywords: Characteristic function; Mixture; Infinite divisibility; Contour integration

\section{Introduction and statement of the theorem}

Recently, the Linnik distribution - originally introduced by Ju.V. Linnik in 1953 (Linnik, 1963) - has attracted attention of a number of researchers (see e.g. Arnold, 1973; Devroye, 1986, 1990; Anderson, 1992;

\footnotetext{
* Corresponding author.
} 
Anderson et al., 1993; Devroye, 1993). Although the characteristic function of this distribution is of a simple form, a general expression of the distribution is not easily attainable. ${ }^{1}$ In this connection, any properties of the distribution such as a mixture representation which facilitates generation of Linnik random variables ought to be of interest. One such property is proved in the present note.

Recall that the generic definition of a Linnik random variable is given in terms of the characteristic function

$$
\varphi_{\alpha}(t)=1 /\left(1+|t|^{\alpha}\right), \quad 0<\alpha<2 .
$$

We shall denote the corresponding density by $p_{\alpha}(x)$. This density can be viewed as a generalization of the well-known Laplace (double exponential) density $p_{2}(x)=c^{-|x|} / 2$ for the case $\alpha=2$ (see, e.g. Johnson and Kotz, 1970). The main result of the paper is the following theorem.

Theorem. For any $0<\alpha<\beta \leqslant 2$, the following equality is valid

$$
\varphi_{\alpha}(t)=\int_{0}^{\infty} \varphi_{\beta}(t / s) g(s ; \alpha, \beta) \mathrm{d} s, \quad-\infty<t<\infty,
$$

where

$$
g(s ; \alpha, \beta)=\left(\frac{\beta}{\pi} \sin \frac{\pi \alpha}{\beta}\right) \frac{s^{\alpha-1}}{1+s^{2 \alpha}+2 s^{\alpha} \cos \frac{\pi \alpha}{\beta}} .
$$

Noting that the equality (1) is equivalent to the following

$$
p_{\alpha}(x)=\int_{0}^{\infty} p_{\beta}(s x) g(s ; \alpha, \beta) \mathrm{d} s, \quad-\infty<x<\infty,
$$

and taking into account that $g(s ; \alpha, \beta)$ is a genuine density function, we arrive at the representation of the form

$$
X_{\alpha} \doteq X_{\beta} Y_{\alpha \beta},
$$

as stipulated in the abstract. This representation allows us to generate Linnik variables of different parameters starting from convenient base, e.g. from the Laplace distribution corresponding to $\beta=2$. That is, our theorem yields immediately the following corollary.

Corollary 1. For any $\alpha \in(0,2)$, Linnik distribution with characteristic function $\varphi_{\alpha}(t)$ is a scale mixture of Laplace distributions with characteristic functions $\varphi_{2}(t / s)=s^{2} /\left(s^{2}+t^{2}\right), 0<s<\infty$.

By Steutel's theorem (Steutel, 1970), any scale mixture of Laplace distributions is infinitely divisible. Therefore, Corollary 1 yields infinite divisibility of Linnik distributions. This fact is not new and was proved in Devroye (1990). However, noting that any mixture of scale mixtures of Laplace distributions is again a scale mixture of Laplace distributions, we obtain a stronger result: any mixture of Linnik distributions is infinitely divisible. More precisely, the following result is valid.

Corollary 2. Let $P$ be a probability measure on the half-strip $S=\{(\alpha, s): 0<\alpha \leqslant 2,0 \leqslant s<\infty\}$. Then the distribution with the characteristic function

$$
\varphi(t)=\int_{s} \varphi_{\alpha}(s t) P(\mathrm{~d} \alpha \mathrm{d} s)
$$

is infinitely divisible.

\footnotetext{
${ }^{1}$ See Hayfavi, A., S. Kotz and I.V. Ostrovskii (1994), Analytic and asymptotic properties of Linnik's probability densities, C.R. Acad. Sci. Paris, Série I, 319, 985-990.
} 


\section{Proof of the theorem}

Note, that the equality (1) is equivalent to the following one:

$$
\frac{1}{1+t^{\alpha}}=\int_{0}^{\infty} \frac{s^{\beta}}{s^{\beta}+t^{\beta}} g(s ; \alpha, \beta) \mathrm{d} s, \quad 0<\alpha<\beta \leqslant 2, t \geqslant 0 .
$$

To prove the latter, we denote

$$
I=\frac{\pi}{\beta \sin \frac{\pi \alpha}{\beta}} \int_{0}^{\infty} \frac{s^{\beta}}{s^{\beta}+t^{\beta}} g(s ; \alpha, \beta) \mathrm{d} s=\int_{0}^{\infty} \frac{s^{\beta+\alpha-1} \mathrm{~d} s}{\left(s^{\beta}+t^{\beta}\right)\left(1+s^{2 \alpha}+2 s^{\alpha} \cos \frac{\pi \alpha}{\beta}\right)} .
$$

It is sufficient to establish that

$$
I=\frac{\pi}{\beta \sin \frac{\pi \alpha}{\beta}} \cdot \frac{1}{1+t^{\alpha}} \text {. }
$$

Transforming the integral $I$ by means of the change of variables $\tau=t^{\beta} s^{-\beta}$, we have

$$
I=\frac{t^{\alpha}}{\beta} \int_{0}^{\infty} \frac{\tau^{(\alpha / \beta)-1} \mathrm{~d} \tau}{(1+\tau)\left(\tau^{2 \alpha / \beta}+t^{2 \alpha}+2 \tau^{\alpha / \beta} t^{\alpha} \cos \frac{\pi \alpha}{\beta}\right)} .
$$

Utilizing the identity

$$
\frac{1}{\tau^{2 \alpha / \beta}+t^{2 \alpha}+2 \tau^{\alpha / \beta} t^{\alpha} \cos \frac{\pi \alpha}{\beta}}=\frac{1}{t^{\alpha} 2 \mathrm{i} \sin \frac{\pi \alpha}{\beta}}\left\{\frac{1}{\tau^{\alpha / \beta}+t^{\alpha} \mathrm{e}^{-\mathrm{i} \pi \alpha / \beta}}-\frac{1}{\tau^{\alpha / \beta}+t^{\alpha} \mathrm{e}^{\mathrm{i} \pi \alpha / \beta}}\right\},
$$

we have

$$
I=\frac{1}{2 \mathrm{i} \beta \sin \frac{\pi \alpha}{\beta}}\left\{\int_{0}^{\infty} \frac{\tau^{(\alpha / \beta)-1} \mathrm{~d} \tau}{(1+\tau)\left(\tau^{\alpha / \beta}+t^{\alpha} \mathrm{e}^{-i \pi \alpha / \beta}\right)}-\int_{0}^{\infty} \frac{\tau^{(\alpha / \beta)-1} \mathrm{~d} \tau}{(1+\tau)\left(\tau^{\alpha / \beta}+t^{\alpha} \mathrm{e}^{\mathrm{i} \pi \alpha / \beta}\right)}\right\} .
$$

Consider the function

$$
q(\tau)=\frac{1}{(1+\tau)\left(\tau^{\alpha / \beta}+t^{\alpha} \mathrm{e}^{i \pi \alpha / \beta}\right)},
$$

in the complex $\tau$-plane cut along the positive ray. Define the branch of $\tau^{\alpha / \beta}$ in (4) by the conditions

$$
\tau^{\alpha / \beta}=r^{\alpha / \beta} \mathrm{e}^{\mathrm{i} \phi \alpha / \beta}, \quad \tau=r \mathrm{e}^{\mathrm{i} \varphi}, \quad 0<\varphi<2 \pi .
$$

Since

$$
\left|\frac{\alpha}{\beta}(\pi-\varphi)\right|<\pi
$$

when $0<\varphi<2 \pi$, we have

$$
\tau^{\alpha / \beta}+t^{\alpha} \mathrm{e}^{\mathrm{i} \pi \alpha / \beta}=\mathrm{e}^{\mathrm{i} \varphi \alpha / \beta}\left(r^{\alpha / \beta}+t^{\alpha} \mathrm{e}^{\mathrm{i} \alpha / \beta(\pi-\varphi)}\right) \neq 0 .
$$

Therefore the function $q(\tau)$ is analytic in the cut out plane. Denote by $G_{R, \varepsilon}$ the simply connected region

$$
G_{R, \varepsilon}=\{\tau: \varepsilon<|\tau|<R\} \backslash\{\tau: \varepsilon<\tau<R\}, 0<\varepsilon<R(>1)
$$

and denote by $\partial G_{R, \varepsilon}$ its boundary traversed in the direction which leaves $G_{R, \varepsilon}$ from the left (the line interval $\{\tau: \varepsilon<\tau<R\}$ is being traversed twice in the opposite directions). By the Cauchy Residue Theorem, we have

$$
\oint_{\tau G_{R, \tau}} q(\tau) \mathrm{d} \tau=2 \pi \mathrm{i}(\text { Residue of } q(\tau) \text { at } \tau=-1)=-\frac{2 \pi \mathrm{i}}{1+t^{\alpha}} .
$$


Taking the limit of the last integral as $R \rightarrow \infty, \varepsilon \rightarrow 0$, we arrive at

$$
\int_{0}^{\infty} \frac{\tau^{(\alpha / \beta)-1} \mathrm{~d} \tau}{(1+\tau)\left(\tau^{\alpha / \beta}+t^{\alpha} \mathrm{e}^{\mathrm{i} \pi \alpha / \beta}\right)}-\mathrm{e}^{2 \pi \mathrm{i}[(\alpha / \beta)-1]} \int_{0}^{\infty} \frac{\tau^{(\alpha / \beta)-1} \mathrm{~d} \tau}{(1+\tau)\left(\tau^{\alpha / \beta} \mathrm{e}^{2 \pi \mathrm{i} \alpha / \beta}+t^{\alpha} \mathrm{e}^{\mathrm{i} \pi \alpha / \beta}\right)}=-\frac{2 \pi \mathrm{i}}{1+t^{\alpha}}
$$

It is evident that this equality can be rewritten in the form

$$
\int_{0}^{\infty} \frac{\tau^{(\alpha / \beta)-1} \mathrm{~d} \tau}{(1+\tau)\left(\tau^{\alpha / \beta}+t^{\alpha} \mathrm{e}^{\mathrm{i} \pi \alpha / \beta}\right)}-\int_{0}^{\infty} \frac{\tau^{(\alpha / \beta)-1} \mathrm{~d} \tau}{(1+\tau)\left(\tau^{\alpha / \beta}+t^{\alpha} \mathrm{e}^{-\mathrm{i} \pi \alpha / \beta}\right)}=-\frac{2 \pi \mathrm{i}}{1+t^{\alpha}} .
$$

Thus, the difference of the integrals appearing in the braces of (3) has been calculated to be equal to $2 \pi \mathrm{i} /\left(1+t^{\alpha}\right)$. Substituting this value into (3), we arrive at (2). The theorem is thus proved.

\section{References}

Anderson, D.N. (1992), A multivariate Linnik distribution, Statist. Probab. Lett. 14, 333-336.

Anderson, D.N. and B.C. Arnold (1993), Linnik distributions and processes, J. Appl. Probab. 30, 330-340.

Arnold, B.C. (1973), Some characterizations of the exponential distribution by geometric compounding, SIAM J. Appl. Math. 24, 242-244

Devroye, L. (1986), Non-Uniform Random Variable Generation (Springer, New York).

Devroye, L. (1990), A note on Linnik's distribution, Statist. Probab. Lett. 9, 305-306.

Devroye, L. (1993), A triptych of discrete distributions related to stable law, Statist. Probab. Lett. 18, 349-351.

Johnson, N.L. and S. Kotz (1970), Distributions in Statistics, Continuous Univariate Distributions, II (J. Wiley, New York).

Linnik, Ju.V. (1963), Linear forms and statistical criteria, I, II, Selected Translations in Math. Statist. Probab. 3, 1-90. (Original paper appeared in: Ukrainskii Mat. Zhournal 5 (1953) 207-290.)

Anderson, D.N. and B.C. Arnold (1993), Linnik distributions and processes, J. Appl. Probab. 30, 330-340.

Steutel, F.W. (1970), Preservation of Infinite Divisibility under Mixing and Related Topics, Vol. 33 (Mathematical Center Tracts, Mathematisch Centrum, Amsterdam). 